\title{
TU/e EmonOWEN

\section{Characterizing cardiorespiratory interaction in preterm infants across sleep states using visibility graph analysis}

Citation for published version (APA):

Zhang, D., Long, X., Xu, L., Werth, J. V. S. W., Wijshoff, R. W. C. G. R., Aarts, R. M., \& Andriessen, P. (2021). Characterizing cardiorespiratory interaction in preterm infants across sleep states using visibility graph analysis. Journal of Applied Physiology, 130(4), 1015-1024. https://doi.org/10.1152/japplphysiol.00333.2020

\section{Document license: \\ TAVERNE}

DOI:

10.1152/japplphysiol.00333.2020

Document status and date:

Published: 01/04/2021

\section{Document Version:}

Publisher's PDF, also known as Version of Record (includes final page, issue and volume numbers)

\section{Please check the document version of this publication:}

- A submitted manuscript is the version of the article upon submission and before peer-review. There can be important differences between the submitted version and the official published version of record. People interested in the research are advised to contact the author for the final version of the publication, or visit the $\mathrm{DOI}$ to the publisher's website.

- The final author version and the galley proof are versions of the publication after peer review.

- The final published version features the final layout of the paper including the volume, issue and page numbers.

Link to publication

\section{General rights}

Copyright and moral rights for the publications made accessible in the public portal are retained by the authors and/or other copyright owners and it is a condition of accessing publications that users recognise and abide by the legal requirements associated with these rights.

- Users may download and print one copy of any publication from the public portal for the purpose of private study or research.

- You may not further distribute the material or use it for any profit-making activity or commercial gain

- You may freely distribute the URL identifying the publication in the public portal.

If the publication is distributed under the terms of Article 25fa of the Dutch Copyright Act, indicated by the "Taverne" license above, please follow below link for the End User Agreement:

www.tue.nl/taverne

Take down policy

If you believe that this document breaches copyright please contact us at:

openaccess@tue.nl

providing details and we will investigate your claim. 


\title{
Characterizing cardiorespiratory interaction in preterm infants across sleep states using visibility graph analysis
}

\author{
(1) Dandan Zhang, ${ }^{1,2}$ (1) Xi Long, ${ }^{1,2}$ Lin Xu, ${ }^{3}$ Jan Werth, ${ }^{1}$ Ralph Wijshoff, ${ }^{2}$ Ronald M. Aarts, ${ }^{1}$ and \\ Peter Andriessen ${ }^{4,5}$ \\ ${ }^{1}$ Department of Electrical Engineering, Eindhoven University of Technology, Eindhoven, The Netherlands; ${ }^{2}$ Philips Research, \\ Eindhoven, The Netherlands; ${ }^{3}$ School of Information Science and Technology, ShanghaiTech University, Shanghai, China; \\ ${ }^{4}$ Department of Neonatology, Máxima Medical Centre, Veldhoven, The Netherlands; and ${ }^{5}$ Department of Applied Physics, \\ Eindhoven University of Technology, Eindhoven, The Netherlands
}

\begin{abstract}
Cardiorespiratory interaction (CRI) has been intensively studied in adult sleep, yet not in preterm infants, in particular across different sleep states including wake (W), active sleep (AS), and quiet sleep (QS). The aim of this study was to quantify the interaction between cardiac and respiratory activities in different sleep states of preterm infants. The postmenstrual age (PMA) of preterm infants was also taken into consideration. The CRI during sleep was analyzed using a visibility graph (VG) method, enabling the nonlinear analysis of $\mathrm{CRI}$ in a complex network. For each sleep state, parameters quantifying various aspects of the CRI characteristics from constructed VG network including mean degree $\left(D_{\mathrm{m}}\right)$ and its variability $\left(D_{\mathrm{sd}}\right)$, clustering coefficient $\left(C C_{\mathrm{m}}\right)$ and its variability $\left(C_{\mathrm{Sd}}\right)$, assortativity coefficient $(\mathrm{AC})$, and complexity $\left(D_{\mathrm{SE}}\right)$ were extracted from the CRI networks. The interaction effect of sleep state and PMA was found to be statistically significant on all CRI parameters except for AC and $D_{\mathrm{SE}}$. The main effect between sleep state and CRI parameters was statistically significant except for $\mathrm{CC}_{\mathrm{m}}$, and that between PMA and CRI parameters was statistically significant except for $D_{\mathrm{SE}}$. In conclusion, the CRI of preterm infants is associated with sleep states and PMA in general. For preterm infants with a larger PMA, CRI has a more clustered pattern during different sleep states, where QS shows a more regular, stratified, and stronger CRI than other states. In the future, these parameters can be potentially used to separate sleep states in preterm infants.
\end{abstract}

NEW \& NOTEWORTHY The interaction between cardiac and respiratory activities is investigated in preterm infant sleep using an advanced nonlinear method (visibility graph) and some important characteristics are shown to be significantly different across sleep states, which has not been studied before.

cardiorespiratory interaction; preterm infant sleep; visibility graph

\section{INTRODUCTION}

Sleep is the most important activity for infants, during which the autonomic nervous system (ANS) and functional neuronal connectivity develop $(1,2)$. For preterm infants, the sleep states are mainly classified as active sleep (AS) and quiet sleep (QS) (3). Sleep states are associated with developmental changes, where the physiological system functions differ across sleep states (4-6). Sleep pattern has been considered as a measure to evaluate the development of the ANS in terms of brain and cardiac maturation in preterm infants. For example, as the frequency component in electroencephalography (EEG) changes over sleep states, monitoring neonatal sleep was taken as a method to understand infants' brain activity (7, 8). Besides, several observations show that heart rate variability (HRV) changes across sleep states $(9,10)$. Therefore, examining HRV in neonates during sleep can provide information on the maturity of the cardiac function in their early postnatal life (9-11).

Cardiorespiratory activity and interaction has been shown to correlate with sleep states due to the regulation of the ANS (12-14). However, there is no universal terminology to define the cardiorespiratory interaction (CRI). For example, Dick et al. (15) used cardiorespiratory coupling (CRC) as a term that illustrates the influences of respiration on heart rate and blood pressure. Joshi et al. (16) used the same term cardiorespiratory coupling as well. In that study, CRC reflects changes in heart rate to respiratory oscillations and coupling from respiratory oscillations to the heart rate. Schäfer et al. (17) defined CRC by cardiorespiratory synchronization in which the heartbeat is synchronized with ventilation. Yasuma and Hayano (18) used respiratory sinus arrhythmia (RSA), a physiological phenomenon that heart rate increases during inspiration and decreases during expiration. Coleman (19) used cardioventilatory coupling (CVC), 
which describes a synchronization between the heart beat and the onset of inspiration. Bartsch et al. (20) used cardiorespiratory phase synchronization (CRPS) to describe phase synchronization of heartbeat intervals and respiratory cycles. Clark et al. (21) described it by a measure of cardiorespiratory interaction (CRI), which does not distinguish between CVC and RSA. With the different approaches borne in mind, we selected the term CRI that was provided by Clark et al. in our study. In this study, the CRI time series was constructed by using the position of each heartbeat to get its respective amplitude of the respiratory signal. According to the definition of CRI, the RSA, as a part of CRI, has been observed in both adults and healthy term infants $(5,22-25)$. However, for preterm infants, some research groups found that the RSA is relatively weak $(26,27)$ and some even could not find RSA $(28,29)$. Besides, CVC also has been investigated in healthy infants, but Mrowka et al. (30) noted that CVC was infrequently observed at any age, which means that CVC might be uneasy and unstable to be detected in preterm infants. Based on these differences, we believe that many aspects (e.g., the nonlinear characteristics) of CRI have not been studied well.

To this end, a visibility graph (VG) method was employed in the present study, as was previously proposed to analyze CRI in adult sleep (12). The VG method constructs a complex network by mapping a time series into a graph, from which several measures can be computed, enabling the characterization of the time series in different aspects, such as periodicity, randomness, and fractalness. For example, a periodic signal, a random signal, and a fractal signal correspond to a regular network, a random network, and a scale-free network, respectively (31). A VG is capable of characterizing both linear and nonlinear properties of a signal and, therefore, is a powerful method to analyze CRI in a wider spectrum beyond RSA. In the past years, the VG method has been employed in the characterization of physiological signals in many applications such as seizure detection with EEG signals (32, 33), epilepsy diagnosis with electrocorticography (34), and spinal cord injury diagnosis (35). However, no studies, to the authors' best knowledge, have investigated the VG features of CRI in preterm infants. This work aims at quantitatively revealing new characteristics of CRI (using the VG method) in different sleep states in preterm infants.

\section{METHODS}

\section{Subjects and Database}

Thirteen preterm infants who admitted to the neonatal intensive care unit (NICU) of the Máxima Medical Centre (MMC), Veldhoven, the Netherlands, were included in this study. The ethical committee of the MMC approved the study and the patients' parents signed an informed consent for this study. The average gestational age (GA), postmenstrual age (PMA), and birth weight of the subjects were $31.0 \pm 2.7 \mathrm{wk}, 33.1 \pm 2.5 \mathrm{wk}$, and $1,751 \pm 524 \mathrm{~g}$, respectively.

The data in this study include the electrocardiography (ECG) signal recorded at $500 \mathrm{~Hz}$ and respiratory signals recorded at $16 \mathrm{~Hz}$ by chest impedance electrodes. According to Prechtl's sleep scoring system (36), sleep states were annotated on 30-s nonoverlapping windows by a trained (pediatric) sleep expert based mainly on videos with the assistance of respiratory data. In this dataset, there were a total of 6,284 epochs $(52.37 \mathrm{~h})$ annotated (QS: $10.98 \%$, AS: $72.88 \%$, Wake (W): $4.63 \%$, Caretaking: $9.60 \%$ and Unknown: $1.91 \%$ ). The Unknown epochs that cannot be precisely annotated (e. g., due to missing data or technical problems of recording videos) and Caretaking epochs (due to the nurses' NICU routine care) were excluded from the analysis. This led to a total of $46.34 \mathrm{~h}$ (5,561 epochs) of data used in this study with an average recording time of $3.56 \pm 1.66 \mathrm{~h}$ for the 13 patients. Table 1 summarizes the distribution of the 30-s sleep state epochs per infant that were included in this study. Moreover, to investigate the relationship between CRI and PMA, we divided these subjects into two PMA groups based on the age with a threshold of $32 \mathrm{wk}$. Subjects with a PMA between $32 \mathrm{wk}$ and $37 \mathrm{wk}$ were in the older group $(n=8)$, and the remaining subjects $(n=5)$ below 32 wk' PMA were in the younger group.

\section{Cardiorespiratory Interaction}

To remove noise from respiratory signals, a 10th-order Butterworth band pass filter $(0.05-2 \mathrm{~Hz})$ was used. After that, we subtracted baseline in the respiratory signal obtained by using a median filter with a 1-s window. For ECG signals, a type II Chebyshev filter $(0.5-120 \mathrm{~Hz})$ was applied to remove the baseline wander. From ECG, heartbeats (R peaks) were located by using an existing QRS detection algorithm (37). After that, for each 30-s epoch, the respiratory data points (amplitude values) at the corresponding time locations of all $\mathrm{R}$ peaks were identified, generating the CRI signal. Figure 1 illustrates the CRI signal obtained from a respiratory signal and the corresponding R-peak sequence. To have a direct visual comparison of CRI signals in different sleep states, we show an exemplary CRI signal in each of the three states in Fig. 2.

\section{VG Analysis}

The VG method proposed by Lacasa et al. (31) was employed to analyze the obtained CRI signal. In general, the VG method maps a signal into a network graph by analyzing the amplitudes of the signal. Given a signal $y_{k}$ and the corresponding time stamp $t_{k}(k=1,2 \ldots n)$, two data points $\left(t_{i}, y_{i}\right)$ and $\left(t_{j}, y_{j}\right)$ can be connected as nodes through an indirect edge, if and only if

$$
\forall k \in(i, j) ; \quad y_{k}<y_{j}-\frac{\left(y_{j}-y_{i}\right)}{\left(t_{j}-t_{i}\right)}\left(t_{j}-t_{k}\right),
$$

where $t_{i}, t_{j}$, and $t_{k}$ denote the location of a point in a time series, $y_{i}, y_{j}$, and $y_{k}$ denote the corresponding point value, and

Table 1. Distribution of the 30-s sleep state epochs per preterm infant included in this study

\begin{tabular}{lcccccc}
\hline \hline & \multicolumn{6}{c}{ States (Epochs) } \\
\cline { 2 - 5 } & AS & QS & W & Caretaking & Unknown & Total Epochs \\
\hline Mean & 352 & 53 & 22 & 46 & 9 & 483 \\
SD & 175 & 49 & 52 & 49 & 12 & 223 \\
Range & $(82-676)$ & $(0-146)$ & $(0-171)$ & $(0-152)$ & $(0-28)$ & $(108-799)$ \\
\hline
\end{tabular}

AS, active sleep; QS, quiet sleep; SD, standard deviation; W, wake. 


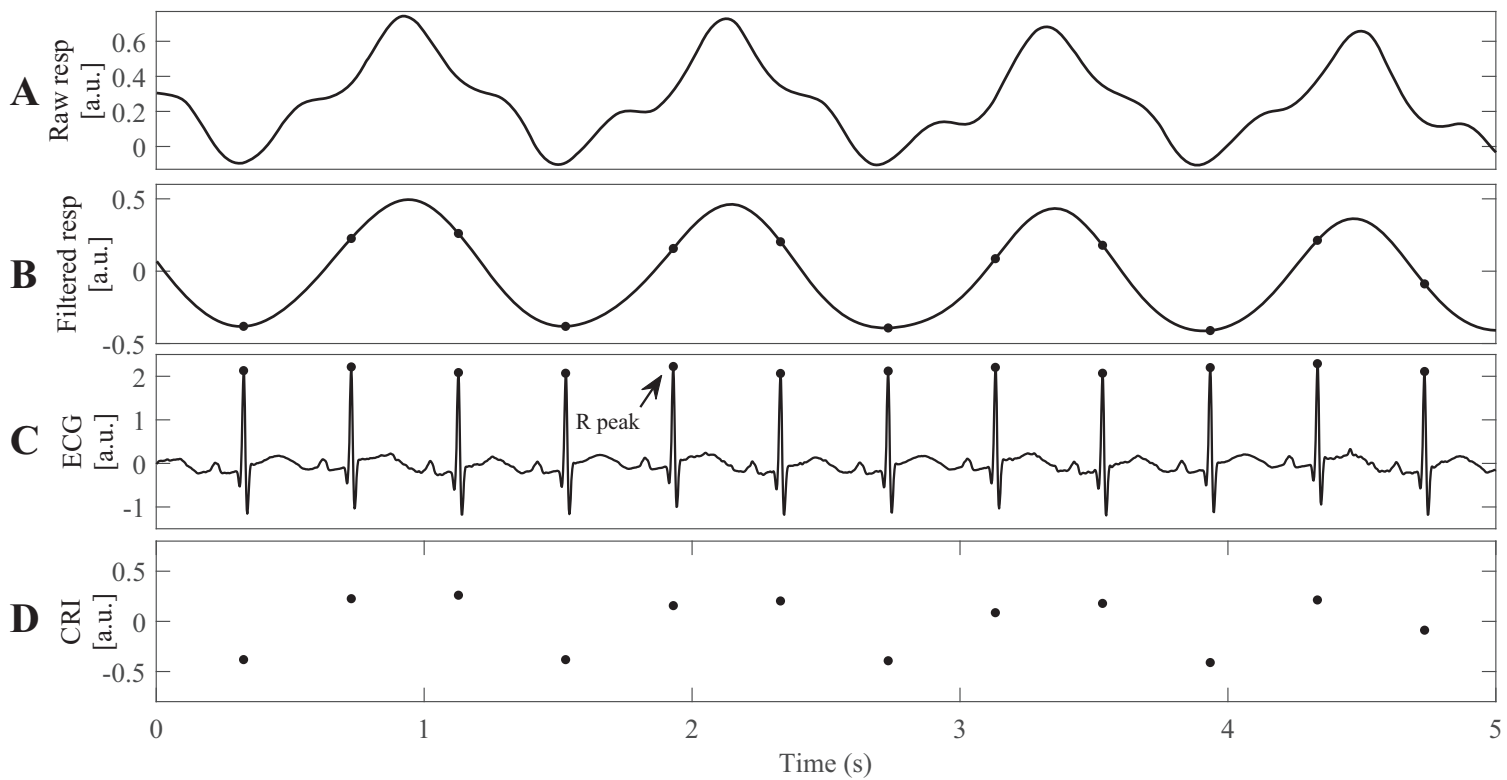

Figure 1. Example of a cardiorespiratory interaction (CRI) signal of a preterm infant, illustrating how the CRI signal is derived from the respiratory and electrocardiography (ECG) signals. A: a raw respiratory signal is filtered with a 10th-order Butterworth band pass filter to obtain a filtered respiratory signal $(B)$. The dots in the filtered respiratory signal are located at the time instants of the R-peaks in the corresponding ECG signal (C). The values of the filtered respiratory signal at the time instants of the corresponding R-peaks constitute the CRI signal $(D)$.

$t_{k}$ is located between $t_{i}$ and $t_{j}$. By applying this rule to all data points of a signal, a network graph is constructed. Figure 3 is an exemplary illustration of the VG network connections of a signal with eight data points (Fig. $3 A$ ) and the corresponding VG network connections (Fig. $3 B$ ). In such a network, the essential condition for point $A$ connecting to point $B$ is that, for any point $C$ between $A$ and $B$, its value should be lower than the data value on the straight line from $A$ to $B$ at the time location of $C$.
Degree $\delta$ is an essential property of a network and it measures the "importance" of a node in this network. The definition of degree is the number of edges attached to a node. For example, as shown in Fig. $3 A$, the degree of nodes $A, B$, and $C$ is 6,3 , and 3 , respectively. Of each epoch, the mean degree $\left(D_{\mathrm{m}}\right)$ and the standard deviation of the degree $\left(D_{\mathrm{sd}}\right)$ were considered in this study. Besides, to understand the regularity pattern of a network, we assessed whether and to what extent the degree distribution $P(\delta)$ follows a power-law

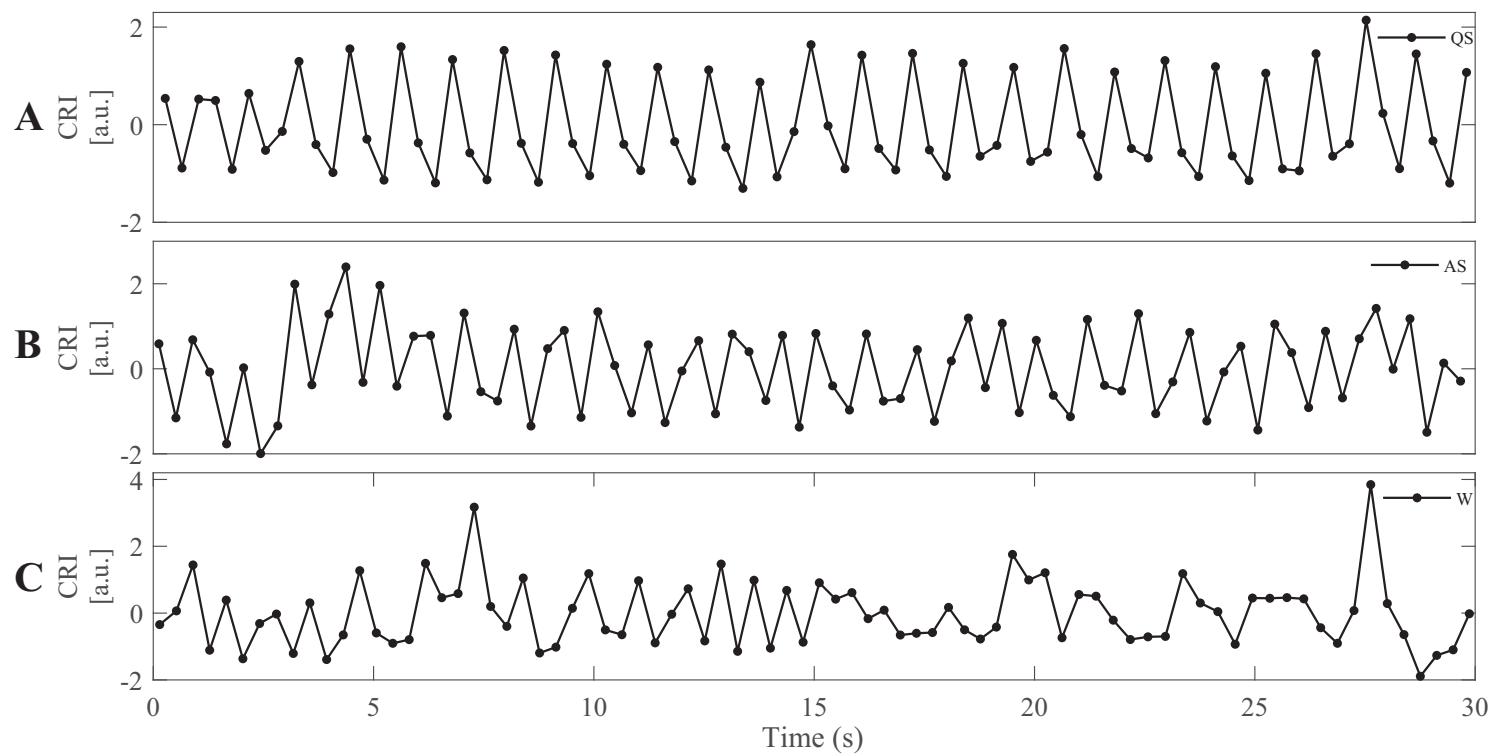

Figure 2. An illustration of normalized cardiorespiratory interaction (CRI) signals during different sleep states. CRI signals have been normalized by dividing them by their respective standard deviations. $A-C$ : correspond to the CRI signals during active sleep (AS) (CRI: $0.02 \pm 0.44)$, quiet sleep (QS) (CRI: $-0.02 \pm 0.18)$, and wake (W) (CRI: $-0.01 \pm 0.46)$, respectively. 

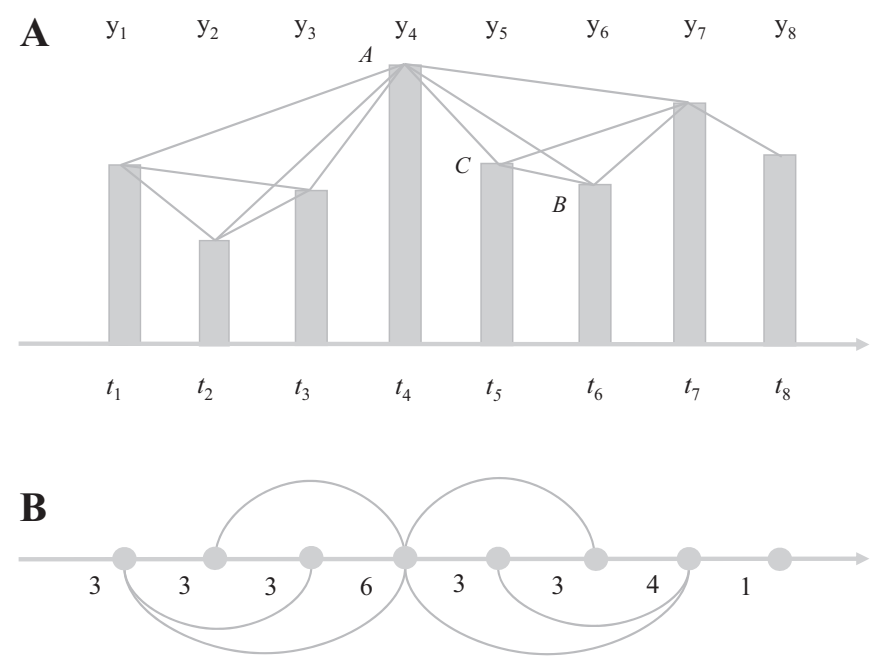

Figure 3. Example of a time series with eight data points $(A)$ and the associated graph derived using the visibility graph (VG) algorithm (B). In graph $(A)$, the vertical bars represent a time series. The data values are given by yi $(i=1,2, \ldots, 8)$ and are displayed above the plot, and the data positions are given by $t_{i}(i=1,2, \ldots, 8)$ and are displayed below the plot. The lines between the data points define the edges connecting the nodes in graph $(A)$. Graph $(B)$ shows the degrees of the nodes of graph $(A)$, where the degree of a node in a network is the number of edges attached to it. Graph $(B)$ shows that the corresponding nodes in graph $(A)$ have degrees $3,3,3,6,3,3,4$, and 1 , respectively.

topology such that $P(\delta) \sim \delta^{-} \lambda$, where the order $\lambda$ indicates the level of regularity $(31,38)$, and a higher regularity corresponds to a larger value of $\lambda$, and vice versa.

The clustering coefficient (CC) measures the density of local clusters in a network (39). For a given node, the CC is defined as the intensity (measured by node degree) of all triangular connections around this node (40), such that

$$
\mathrm{CC}=\frac{N_{v}}{k_{v}\left(k_{v}-1\right) / 2},
$$

where $v$ represent the $v$ th node, $k_{v}$ is the corresponding degree, and $N_{v}$ is the number of links between neighbors of $v$. The CC of a node $v$ reflects the possibility that nodes adjacent to node $v$ are also adjacent to each other. As an extension, we define $\mathrm{CC}_{\mathrm{m}}$ as the average of $\mathrm{CC}$ over all nodes, which measures the cliquishness of a typical fraction and reflects the quantification of a network integration capacity (41). A greater $\mathrm{CC}_{\mathrm{m}}$ value indicates that, in a network, nodes tend to be "integrated" more in a cluster. Besides, we also explored the standard deviation of CC values, indicated by $\mathrm{CC}_{\mathrm{sd}}$, where more clustered nodes are expected to have a lower variability in CC, whereas less clustered nodes are expected to have a higher variability.

A network is considered assortatively mixed, if nodes with similar values of a scalar characteristic tend to be connected together more often than those with different values of that characteristic. For example, in a network that shows assortative mixing by degree, the high-degree nodes will be preferably connected to other high-degree nodes and the same holds for low-degree nodes too (42). Assortative mixing by degree can be measured by the assortativity coefficient (AC) (42), which represents the skewness of node connections. This measure has been utilized in adult sleep research (12), seizure pattern analysis (33), and social networks (43). The computation of $\mathrm{AC}$ is expressed by

$$
\mathrm{AC}=\frac{M^{-1} \sum_{i} \alpha_{i} \beta_{i}-\left[M^{-1} \sum_{i} \frac{1}{2}\left(\alpha_{i}+\beta_{i}\right)\right]^{2}}{M^{-1} \sum_{i} \frac{1}{2}\left(\alpha_{i}^{2}+\beta_{i}^{2}\right)^{2}-\left[M^{-1} \sum_{i} \frac{1}{2}\left(\alpha_{i}+\beta_{i}\right)\right]^{2}},
$$

where $M$ is the total number of edges in a network, and $\alpha_{i}$ and $\beta_{i}$ are the degrees of the two nodes that connect the $i$ th edge. The value of $\mathrm{AC}$ is between -1 and 1 . An AC greater than zero means that the network is mixed with assortativity. An AC equal to zero implies that the network connections between high- and low-degree nodes are random. An AC smaller than zero indicates the network is mixed with disassortativity, i.e., connections exist more likely between high- and low-degree nodes. To measure the extent of skewness of the node connections in a CRI network, AC was inspected in this study.

The sample entropy (SE) is a quantification of complexity and is widely used for assessing the complexity of a biological signal $(44,45)$. Lucchini et al. (46) suggested that SE describes the preterm infants' ANS that directly links to sleep states. Werth et al. (47) used it in preterm infant sleep research. Here, we explored the SE of a CRI network across different sleep states. A signal with a lower SE value carries less information, shows lower complexity and more regularity. A signal with a higher SE value carries more information, shows a higher complexity and less regularity. Here, we investigated the SE of the degree of the nodes $\left(D_{\mathrm{SE}}\right)$ in a CRI network. Assume that we have a series of node degrees with length $N=\left\{x_{1}, x_{2}, \ldots, x_{N}\right\}$ at the corresponding time stamp $1,2, N$, then we define a template vector of length $m$, such that $X_{m}(i)=\left\{x_{i}, x_{i}+1, \ldots, x_{i+m-1}\right\}$ and the distance function $d\left[X_{m}(i), X_{m}(j)\right](i \neq j)$, then we computed as such that

$$
D_{\mathrm{SE}}=-\log A / B
$$

where $A$ is the number of template vector pairs having $d\left[X_{m+1}(i), X_{m}+{ }_{1}(j)\right]$ smaller than tolerance $r$, and $B$ is the number of template vector pairs having $d\left[X_{m}(i), X_{m}(j)\right]$ smaller than $r$. In this study, we calculated $D_{\mathrm{SE}}$ by taking the value of $m$ to be 2 and tolerance $r$ to be $0.2 \times$ std. Where std stands for standard deviation that be taken over the whole degree series.

\section{Statistical Analysis}

Although the aim of this study was to investigate the association between sleep states and CRI, it is important to consider PMA as a potential confounding factor. Before statistical analysis, we tested all variables for normality with the Kolmogorov-Smirnov test and the homogeneity of group variances with the Levene's test. Depending on the test results of normality and homogeneity of variances, the Scheirer-Ray-Hare test, which is an equivalence of two-way ANOVA, was performed. Two main effects (i.e., sleep state and PMA) and the interaction effect between sleep state and PMA were considered. In addition, Dunn tests were applied to identify which pairs of sleep states are different, if the results of the Scheirer-Ray-Hare test were statistically significant. Here, $P<0.05$ was considered significant and Bonferroni correction was used to adjust the $P$ value. 


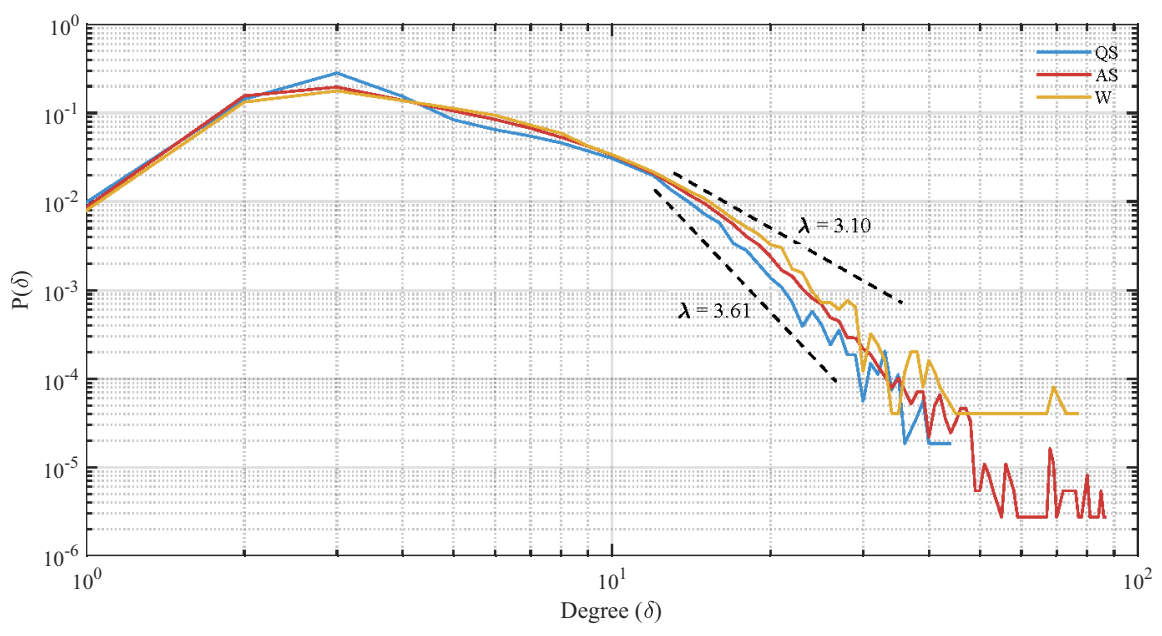

Figure 4. Log-log plot of degree distribution $P(\delta)$ of cardiorespiratory interaction (CRI) over degree during quiet sleep (QS), active sleep (AS), and wake (W). $P(\delta)$ and $\delta$ follow a power-law relationship $P(\delta)$ $\sim \delta^{-\lambda}$ with $\lambda$ of 3.61 for QS, 3.46 for AS, and 3.10 for $W$. The separation between QS, AS, and W can be observed at degree larger than approximately 13.

\section{RESULTS}

To show the overall distribution of degrees, we pooled all the epochs for each sleep state. For clarity, we present our results following the order that describes the characteristics of the CRI-VG network in the above. The degree distribution $P(\delta)$ of the CRI-VG networks in QS, AS, and W versus degree $\delta$ is shown in Fig. 4. We can see in the figure that the distribution of degree follows a power-law topology for all sleep states especially for degree larger than 13, and the exponent $\lambda$ varied across states (QS: $\lambda=3.61$, AS: $\lambda=3.46, \mathrm{~W}: \lambda=3.10$ ). The results of the Scheirer-Ray-Hare test are summarized in Table 2. Besides, Tables 3 and 4 summarize the median and interquartile range (IQR) results of the parameters (representing different aspects of CRI characteristics) in the three sleep states and PMA groups, respectively. Furthermore, Fig. 5 presents the boxplots of the CRI parameters in different sleep states and PMA groups.

There was a statistically significant interaction effect between sleep state and PMA on $D_{\mathrm{m}}[H(\mathrm{df}=2)=40.402, P<$ 0.001 ] as shown in Table 2. The main effect analysis showed that $D_{\mathrm{m}}$ was statistically significant on both sleep state $[H$ $(\mathrm{df}=2)=304.272, P<0.001]$ and PMA group $[H(\mathrm{df}=1)=$ 24.239, $P<0.001$ ]. Figure $5 A$ illustrates that $D_{\mathrm{m}}$ in the younger group was slightly larger than in the older group in case of QS and AS, and that $D_{\mathrm{m}}$ was slightly smaller in the younger group than in the older group in case of W. In addition, for $D_{\text {sd }}$, the main and interaction effects were all found to be statistically significant, with a slightly larger $D_{\text {sd }}$ value in the younger group than in the older group in case of QS and AS, and a slightly smaller $D_{\text {sd }}$ value in the younger group than in the older group in case of W (see Fig. 5B).

For network clustering characteristics, the interaction effect on $\mathrm{CC}_{\mathrm{m}}$ was also statistically significant $[H(\mathrm{df}=2)=$ 33.291, $P<0.001$ ]. The main effect of PMA exhibited statistically significant difference $[H(\mathrm{df}=1)=140.624, P<0.001]$, but there was no statistically significant difference found in different sleep states $[H(\mathrm{df}=2)=4.372, P=0.112]$. Figure $5 \mathrm{C}$ shows that $\mathrm{CC}_{\mathrm{m}}$ in the older group was slightly larger than in the younger group for QS $(P<0.001)$ and AS $(P<0.001)$. However, there was no significant difference between the two age groups for $\mathrm{W}(P=0.14)$. For $\mathrm{CC}_{\mathrm{sd}}$, we found a statistically significant interaction between sleep state and PMA $[H$ $(\mathrm{df}=2)=73.263, P<0.001]$, as well as the independent main effect from sleep state $[H(\mathrm{df}=241.044, P<0.001)]$ and PMA $[H(\mathrm{df}=1)=61.428, P<0.001]$. The younger group seemed to correspond to a smaller $\mathrm{CC}_{\mathrm{sd}}$, in case of QS and AS. In case of $\mathrm{W}$, the younger group seemed to correspond to a larger $\mathrm{CC}_{\mathrm{sd}}$.

For AC, there was no statistically significant interaction effect found between sleep state and PMA $[H(\mathrm{df}=2)=3.996$, $P=0.08]$. Therefore, we did not perform further post hoc test on significance of difference between PMA groups in different sleep states, although the boxplots are presented in Fig. $5 E$. However, statistically significant difference was found between sleep states $[H(\mathrm{df}=2)=107.391, P<0.001]$ and between PMA groups $[H(\mathrm{df}=1)=42.748, P<0.001]$. Dunn post hoc test revealed a significant difference between QS and AS $(P<0.001)$ and between QS and W $(P<0.001)$, but

Table 2. Results from the Scheirer-Ray-Hare test examining CRI parameters across sleep states and PMA

\begin{tabular}{|c|c|c|c|c|c|c|c|c|c|}
\hline$\overline{D_{\mathrm{m}}}$ & 2 & 304.272 & $<0.0001$ & 1 & 24.239 & $<0.0001$ & 2 & 40.402 & $<0.0001$ \\
\hline $\mathrm{CC}_{\mathrm{m}}$ & 2 & 4.372 & + & 1 & 140.624 & $<0.0001$ & 2 & 33.291 & $<0.0001$ \\
\hline $\mathrm{CC}_{\mathrm{sd}}$ & 2 & 241.044 & $<0.0001$ & 1 & 61.428 & $<0.0001$ & 2 & 73.263 & $<0.0001$ \\
\hline$A C$ & 2 & 107.391 & $<0.0001$ & 1 & 42.748 & $<0.0001$ & 2 & 3.996 & + \\
\hline$D_{\mathrm{SE}}$ & 2 & 267.333 & $<0.0001$ & 1 & 2.604 & + & 2 & 2.287 & + \\
\hline
\end{tabular}

AC, assortativity coefficient; $\mathrm{CC}_{\mathrm{m}}$, mean clustering coefficient; $\mathrm{CC}_{\mathrm{sd}}$, standard deviation of clustering coefficient; CRI, cardiorespiratory interaction; $D_{\mathrm{m}}$, mean degree; $D_{\mathrm{sd}}$, standard deviation of the degree; $D_{\mathrm{SE}}$, sample entropy of the degree of the nodes; df, degree of freedom; $H$, nonparametric Scheirer-Ray-Hare statistic; PMA, postmenstrual age. TThere is no significant difference. 
Table 3. Median, IQR, and significance analysis of CRI parameters in sleep states

\begin{tabular}{|c|c|c|c|c|c|c|}
\hline & QS & AS & w & QS vs. AS & \multicolumn{2}{|c|}{$P$ Value } \\
\hline$D_{\mathrm{m}}$ & $5.013(4.744-5.605)$ & $5.579(5.092-6.216)$ & $5.865(5.372-6.636)$ & $<0.001$ & $<0.001$ & $<0.001$ \\
\hline $\mathrm{CC}_{\mathrm{m}}$ & $0.761(0.744-0.777)$ & $0.762(0.748-0.776)$ & 0.759 (0.749-0.771) & $\ddagger$ & $\ddagger$ & $\ddagger$ \\
\hline $\mathrm{CC}_{\mathrm{sd}}$ & $0.269(0.257-0.280)$ & $0.259(0.244-0.272)$ & $0.250(0.233-0.262)$ & $<0.001$ & $<0.001$ & $<0.001$ \\
\hline$A C$ & $0.092(0.048-0.140)$ & $0.063(0.016-0.110)$ & $0.058(0.020-0.104)$ & $<0.001$ & $<0.001$ & + \\
\hline$D_{\mathrm{SE}}$ & $1.494(1.300-1.756)$ & $1.758(1.459-2.104)$ & $1.981(1.613-2.392)$ & $<0.001$ & $<0.001$ & $<0.001$ \\
\hline
\end{tabular}

Results are presented as median (25th-75th percentile). Dunn test was applied here to examine the difference between each two states. AC, assortativity coefficient; AS, active sleep; $\mathrm{CC}_{\mathrm{m}}$, mean clustering coefficient; $\mathrm{CC}_{\mathrm{sd}}$, standard deviation of clustering coefficient; CRI, cardiorespiratory interaction; $D_{\mathrm{m}}$, mean degree; $D_{\mathrm{sd}}$, standard deviation of the degree; $D_{\mathrm{SE}}$, sample entropy of the degree of the nodes; IQR, interquartile range; QS, quiet sleep; W, wake. †There is no significant difference. ₹Post hoc test was not applicable (not necessarily performed) where no significant difference between the three states was found after Scheirer-Ray-Hare test.

no statistically significant difference between AS and $\mathrm{W}(P=$ 0.78).

The interaction effect on $D_{\mathrm{SE}}$ was not statistically significant $[H(\mathrm{df}=2)=2.287, P=0.248]$ as suggested by the results in Table 2. Similar to AC, post hoc test was not applicable. Concerning the main effects, only the sleep state effect was found to be statistically significant $[H(\mathrm{df}=2)=267.333, P<$ 0.001]. Therefore, Dunn's post hoc test was performed solely on verifying the significance of difference between each two sleep states, where significant differences were found.

\section{DISCUSSION}

In this study, CRI during different sleep states was investigated in preterm infants. Based on the VG-method, the CRI during sleep was quantified by mapping it into a network. Several parameters were then extracted from the CRI network, which, in general, have been shown to be different for different sleep states, indicating the existence of CRI in preterm infants during sleep and revealing the extent of CRI through changes in sleep states. In addition, the effect of the confounding factor PMA was analyzed as well as the interaction effect of sleep state and PMA on these CRI parameters, which revealed that some characteristics of CRI in different sleep states depended on PMA.

Table 4. Median, IQR, and significance analysis of $C R I$ parameters in PMA groups

\begin{tabular}{llcc}
\hline \hline & & & \multicolumn{1}{c}{$\boldsymbol{P}$ Value } \\
\cline { 3 - 4 } & \multicolumn{1}{c}{ Younger Group } & Older Group & $\begin{array}{c}\text { Younger group vs. } \\
\text { Older group }\end{array}$ \\
\hline$D_{\mathrm{m}}$ & $5.604(5.128-6.189)$ & $5.425(4.962-6.173)$ & $<0.0001$ \\
$D_{\text {sd }}$ & $3.688(3.285-4.243)$ & $3.565(3.205-4.105)$ & $<0.0001$ \\
$C_{m}$ & $0.758(0.745-0.771)$ & $0.765(0.751-0.779)$ & $<0.0001$ \\
$C C_{\text {sd }}$ & $0.257(0.244-0.270)$ & $0.263(0.246-0.275)$ & $<0.0001$ \\
$A C$ & $0.073(0.021-0.126)$ & $0.061(0.018-0.121)$ & $<0.0001$ \\
$D_{\text {SE }}$ & $1.749(1.447-2.094)$ & $1.716(1.421-2.067)$ & $\ddagger$ \\
\hline
\end{tabular}

Results are presented as median (25th-75th percentile). Dunn test was applied here to examine the difference between the two groups. $\mathrm{AC}$, assortativity coefficient; $\mathrm{CC}_{\mathrm{m}}$, mean clustering coefficient; $\mathrm{CC}_{\mathrm{sd}}$, standard deviation of clustering coefficient; CRI, cardiorespiratory interaction; $D_{\mathrm{m}}$, mean degree; $D_{\mathrm{sd}}$, standard deviation of the degree; $D_{\mathrm{SE}}$, sample entropy of the degree of the nodes; IQR, interquartile range; PMA, postmenstrual age. \#Post hoc test was not applicable (not necessarily performed) where no significant difference between the PMA groups was found after Scheirer-Ray-Hare test.
For $P(\delta)$ of CRI in preterm infants, Fig. 4 shows that it follows a power-law relationship for QS, AS, and W. One can distinguish between different sleep states for $\delta$ larger than 13. These results show that the CRI signal is a fractal series, which was supported by the findings by Song et al. (48) who showed that power-law degree distributions are related to fractality in a physiological signal network. Based on the characteristics of fractal series that their network structures should be similar on all scales, we can speculate that CRI has similar structure during different sleep states. This might relate to phase synchronization of heartbeat intervals and respiratory cycles in CRPS (20). This means that VG is a useful method to reveal the underlying information from the interaction between cardiac and respiratory activity. Moreover, the $P(\delta)$ in QS was more narrow in comparison with the other two states (especially for $\delta$ from 5 to 30 ) which exhibits that CRI is more regular during QS than AS. Similar results were found by Long et al. (12) in adult sleep research. Actually, the fractal property also exists in respiratory variability and hear rate variability (HRV). For respiratory variability, the interbreath interval shows fractal complexity and appears degraded with increasing age in adult respiratory research (49). For HRV, the power-law is considered a quantitative analysis of the power spectrum in the region of the ultralow $\left(10^{-4} \mathrm{~Hz}\right)$ and very-low frequency $\left(10^{-2} \mathrm{~Hz}\right)$ bands, and the fractal scaling exponent reflects fractal-like scaling characteristics of HRV (50). However, that method is unavailable to measure RSA, as RSA locates in the high-frequency band of HRV.

The significant effect of sleep state and PMA on $D_{\mathrm{m}}$ and $D_{\text {sd }}$ suggests that CRI during different sleep states depends on the age of preterm infants. Moreover, in the older group, we observed lower values of $D_{\mathrm{m}}$ and $D_{\mathrm{sd}}$ in QS as compared with AS. This indicates that the CRI network has more connections with a higher variability during AS than during QS. One explanation is that CRI was stronger during QS than AS. Similar results can be found in adult sleep research work by Long et al. (12), which strengthens the validity of our results. In addition, the values of $D_{\mathrm{m}}$ and $D_{\mathrm{sd}}$ were smaller (i.e., the CRI was more stable) for the older group than those for the younger group in QS and AS. An explanation might be attributed to the fact that the heart-lung interaction or function is not well developed in very preterm infants (51) and will become more mature with increased age.

For $\mathrm{CC}_{\mathrm{m}}$, although no significant difference was found between sleep states, the existence of significance of the 

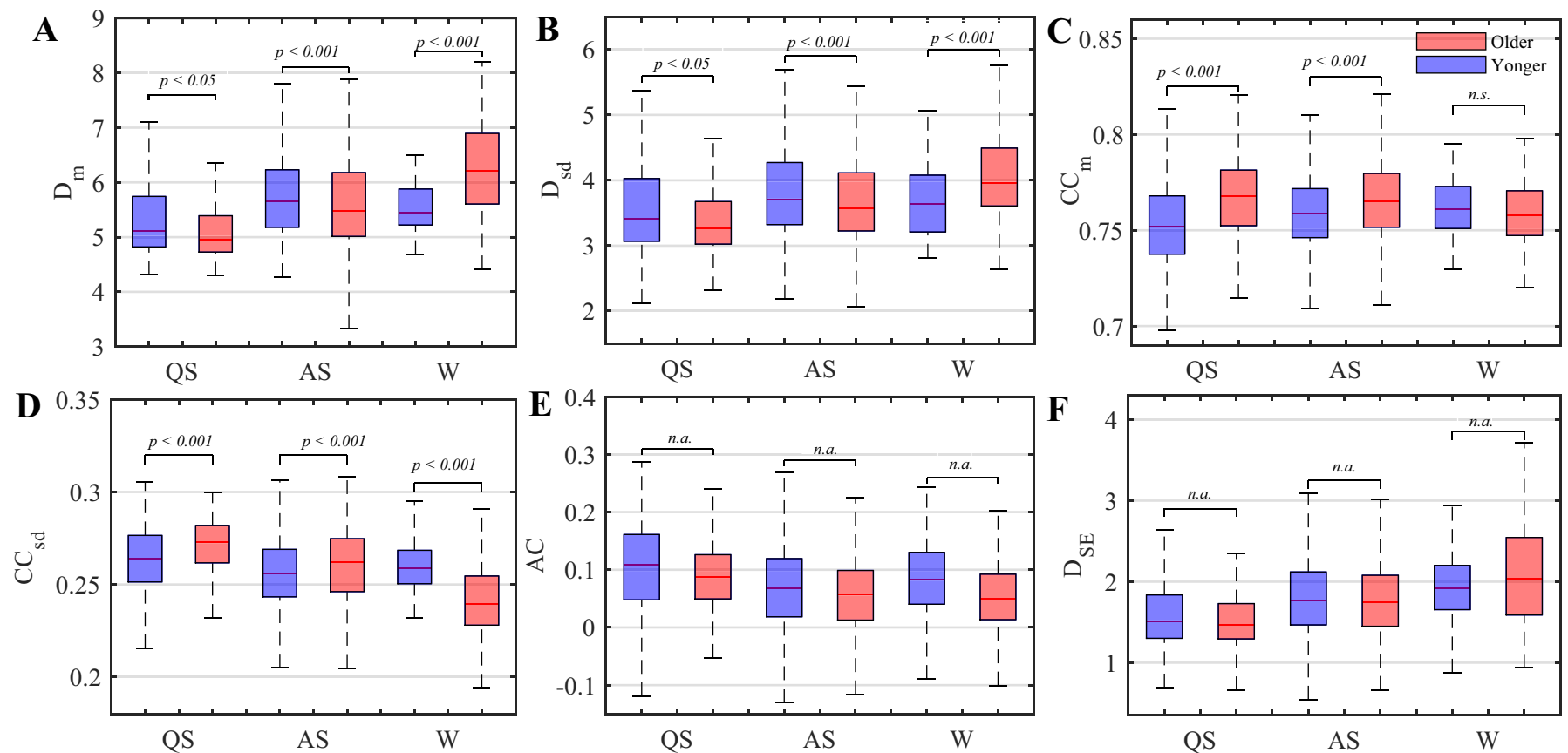

Figure 5. The comparison of the cardiorespiratory interaction-visibility graph (CRI-VG) parameters for each postmenstrual age (PMA) group [Younger $(n=5)$ : PMA < $32 \mathrm{wk}$; Older $(n=8): 32 \leq \mathrm{PMA}<37 \mathrm{wk}$ in different sleep states. $A-F$ : present the comparison of Dm, Dsd, CCm, CCsd, AC and DSE in different sleep states, respectively. AC, assortativity coefficient; CCm, mean clustering coefficient; CCsd, standard deviation of clustering coefficient; CRI, cardiorespiratory interaction; Dm, mean degree; Dsd, standard deviation of the degree; DSE, sample entropy of the degree of the nodes; Dunn test was applied here to examine the statistical difference between age group in different sleep states. n.s., there is no significant difference; n.a., not applicable (not necessarily performed). Note that, for better readability, outliers were removed in these plots.

interaction effect of PMA suggests the dependency of the cluster integration capacity of CRI networks on PMA. The clustering ability in older group is higher than in the younger group. According to network theory, hubs (nodes with high degree) usually exhibit a lower local clustering (with a smaller clustering coefficient) and therefore a weak network (52). This indicates that the CRI in preterm infants is stronger and firmer in older infants than in younger ones. We also anticipate that the network cluster integration of CRI would increase along with the increase of age, which is consistent with the previous finding by Clark et al. (21). Interestingly, as shown in Fig. $5 C, C_{m}$ showed significant difference between the two age groups in QS and AS but not in W. This means that the significant interaction effect was mainly explained or contributed by the age difference in QS and AS rather than in W. Actually, for both age groups, the cardiac (ECG) and respiratory measurements in $\mathrm{W}$ could be interfered by motion artifacts due to body movements. Furthermore, we also think the characteristics of CC might associate with RSA to some extent. If there exists RSA, the slope of inspiration has a larger possibility to occur in the constructed CRI time series, as the heart rate increases during inspiration, resulting in the inspiratory part of the respiratory signal to be sampled more densely. Based on the characteristics of $\mathrm{CC}_{\mathrm{m}}$, this should result in local clustering in the CRI network. Combined with the result of Fig. $5 C$, we can speculate that there exists slight RSA in the preterm infants. This result is consistent with the findings by Patzak et al. (26). As for $\mathrm{CC}_{\text {sd }}$, we see in Fig. $5 D$ that QS corresponded to a larger variability in CC than AS. In comparison with the younger group, the older group has a larger $\mathrm{CC}_{\mathrm{sd}}$ in QS and $\mathrm{AS} . \mathrm{CC}_{\mathrm{sd}}$ shows the amount of variation in CC, where less variation in CC means a weaker CRI. Those results depict that CRI expresses stronger in QS than in AS and stronger in the older group than in the younger group during QS and AS. These results are consistent with our speculative conclusion of the analysis of $\mathrm{CC}_{\mathrm{m}}$.

The absence of significant interaction between sleep states and PMA on AC means the assortativity of CRI networks does not depend on PMA. The value of AC in all the sleep states varies around zero, but with a larger positive portion than a negative portion. This indicates that CRI networks can be disassortative, random, and assortative, but mostly present some assortativity. Moreover, Fig. $5 E$ depicts that QS on average shows a larger assortativity than AS. One possible explanation could be that CRI during QS exhibits a more regular and stronger pattern than during AS, which is very comparable to work performed in adult sleep research (12). The assortativity in younger group is slightly higher than the older group. This result is controversial with our anticipation. The reason is unclear and one possible reason could be that the assortative networks are more robust (42), which causes slight differences between younger group and older group. To prove this speculation, more subjects should be investigated in the future. Based on analysis on AC, we can conclude that the CRI networks show a stratified structure during sleep states. This phenomenon might associate with RSA, because if there exists RSA, most nodes in the CRI network will correspond to inspiration. Hubs will have a larger possibility to present the peaks in respiration. The CRI network shows associative mixing most of the time across the different sleep states; this result might support our assumption here. One disturbance factor to this assumption is that 
artifacts caused by motion also have a large possibility to appear as hubs in the CRI network, but the assortativity of a network is not sensitive to artifacts. In combination with the result that there is no significant difference between AS and $\mathrm{W}$, we can speculate that AC in CRI network is partially related with RSA.

For $D_{\mathrm{SE}}$, significant difference was only found between sleep states. The complexity of CRI network in W is significantly larger than in AS, followed by QS. This result could be explained by CRI being strongest in QS, and the CRI network to be sensitive to motion artifacts in AS and W. However, this result was different from the findings in a previous study by Lucchini et al. (53), where they performed entropy analysis in cardiorespiratory dynamics and found higher entropy measures in QS than other sleep states in term infants. This difference could be due to the decreased parasympathetic and increased sympathetic activity $(54,55)$ in preterm infants. Another possible reason could be that preterm infants have a less complex heart rate variability than term infants (13). There is no significant difference in $D_{\mathrm{SE}}$ between the age groups. $D_{\mathrm{SE}}$ should be further investigated in a larger data set from more (preterm) infants with a wider range of age, to conclude on any possible differences.

The conventional methods to analyze CRI are based on time and frequency domain analyses, where one measures the magnitude of the fluctuations around the mean in the CRI time series or in some predetermined frequency bands. For example, Lucchini et al. (56) explored the cardiorespiratory phase synchronization and directionality in infants by frequency analysis; Scher et al. (57) used the spectral measures to investigate cardiorespiratory behavior in neonates during sleep. It has a potential limitation that they need to set a frequency threshold before using their method. It might miss some useful information in this case, if the frequency threshold is not selected properly. Compared with those conventional methods, VG provides an alternative approach that focuses on the structure of time series to explore and to quantify the underlying information. In this study, we only measured several basic characteristics of the CRI network. We can readily find that the VG can help us explore clusters in the time series (by using the clustering coefficient), potential preferential selection, and stability of the structure in the time-series (by using the assortativity coefficient), and observe the potential recurrence patterns in the time series (by investigating the degree distribution). More properties of the CRI network are still to be explored, such as the distribution of $\mathrm{CC}_{\mathrm{m}}$ over the degree, which reveals the modularity of a network (58). Moreover, compared with those conventional methods, the VG network is more robust, as the VG focuses on the structure of time series, which means the VG is not sensitive to the amplitude fluctuation of a time series. The VG also has limitations though. One limitation is that it does not distinguish the direction of regulation in cardiorespiratory interaction. Transfer entropy would be a method that can show directionality in the regulation (53). Also, we can explore the CRI network further by using directed networks which take into consideration the direction of time series and weight on the edges (59).

Finally, in comparison with traditional measurements, such as polysomnography (PSG), which measures multi- channel electrophysiology signals by attaching many electrodes on the skin of the subject, the cardiorespiratory activity can be monitored in an unobtrusive way (60) with reduced risks of damaging the fragile skin of infants and disturbing their sleep. Moreover, the respiratory and ECG signals can be reliably and easily acquired in a noncontact way, using, e.g., camera (61), radar $(62,63)$, or a capacitive mattress (64). Based on our study, we found that the VG-based CRI characteristics are different across sleep states. That means these measures may be employed to distinguish sleep states in preterm infants in an unobtrusive manner. In the future, we will apply the CRI network in unobtrusive monitoring of the preterm infants' sleep states.

\section{CONCLUSIONS}

In summary, the cardiorespiratory interaction in preterm infants was quantified by the visibility graph method, a technique to analyze the nonlinearity of CRI in a two-dimensional complex network. Our results suggest that the complexity of the CRI is a highly useful index for sleep state research in preterm infants. Potentially, quantifying CRI by a visual network provides a useful method to estimate the maturation of the autonomic regulatory system and the development of the cardiorespiratory activity (like CRPS and RSA) in preterm infants. Moreover, in the future, CRI networks can provide some new characteristics to separate the sleep states of the preterm infant in unobtrusive preterm infant sleep research.

\section{ACKNOWLEDGMENTS}

The authors thank Xuan Zhou and Zhenghui Wen for comments and suggestions, and Mohammed Meftah who contributed to data collection, as well as the TU/e colleagues in the sleep research group who provided insightful inspirations and comments.

\section{GRANTS}

The Eindhoven MedTech Innovation Center (e/MTIC) and the China Scholarship Council (CSC) (Grant No. 201806170049) supported this work.

\section{DISCLOSURES}

No conflicts of interest, financial or otherwise, are declared by the authors.

\section{AUTHOR CONTRIBUTIONS}

X.L. and R.M.A. conceived and designed research; P.A. performed experiments; D.Z. analyzed data; D.Z., X.L., and P.A. interpreted results of experiments; D.Z. prepared figures; D.Z. and X.L. drafted manuscript; D.Z., X.L., L.X., J.W., R.W., R.M.A., and P.A. edited and revised manuscript; D.Z., L.X., J.W., R.W., R.M.A., and P.A. approved final version of manuscript.

\section{REFERENCES}

1. Ludington-Hoe SM, Johnson MW, Morgan K, Lewis T, Gutman J, Wilson PD, Scher MS. Neurophysiologic assessment of neonatal sleep organization: preliminary results of a randomized, controlled 
trial of skin contact with preterm infants. Pediatrics 117: e909-e923, 2006. doi:10.1542/peds.2004-1422.

2. Thoman EB, Ingersoll EW. Sleep/wake states of preterm infants: stability, developmental change, diurnal variation, and relation with caregiving activity. Child Dev 70: 1-10, 1999. doi:10.1111/1467. 8624.00001.

3. Parmelee AH, Wenner WH, Akiyama Y, Schultz M, Stern E. Sleep states in premature and full-term newborn infants. Dev Med Child Neuro/ 9: 70-77, 1967. doi:10.1111/j.1469-8749.1967.tb02212.x.

4. Foreman SW, Thomas KA, Blackburn ST. Individual and gender differences matter in preterm infant state development. J Obstet Gynecol neonatal Nurs 37: 657-665, 2008. doi:10.1111/j.15526909.2008.00292.x.

5. Holditch-Davis D, Scher M, Schwartz T, Hudson-Barr D. Sleeping and waking state development in preterm infants. Early Hum Dev 80: 43-64, 2004. doi:10.1016/j.earlhumdev.2004.05.006.

6. Orem J. Medullary respiratory neuron activity: relationship to tonic and phasic REM sleep. J Appl Physiol Respir Environ Exerc Physiol 48: 54-65, 1980. doi:10.1152/jappl.1980.48.1.54.

7. Dereymaeker A, Pillay K, Vervisch J, De Vos M, Van Huffel S, Jansen K, Naulaers G. Review of sleep-EEG in preterm and term neonates. Early Hum Dev 113: 87-103, 2017. doi:10.1016/j.earlhumdev. 2017.07.003

8. Janjarasjitt S, Scher MS, Loparo KA. Nonlinear dynamical analysis of the neonatal EEG time series: the relationship between sleep state and complexity. Clin Neurophysiol 119: 1812-1823, 2008. doi:10.1016/j.clinph.2008.03.024.

9. Javorka K, Lehotska Z, Kozar M, Uhrikova Z, Kolarovszki B, Javorka M, Zibolen $\mathbf{M}$. Heart rate variability in newborns[Online]. Physiol Res 66: S203-S214, 2017. doi:10.33549/physiolres.933676.

10. Vandeput S, Naulaers G, Daniels H, Van Huffel S. Heart rate variability during REM and non-REM sleep in preterm neonates with and without abnormal cardiorespiratory events. Early Hum Dev 85: 665671, 2009. doi:10.1016/j.earlhumdev.2009.09.007.

11. Doyle OM, Korotchikova I, Lightbody G, Marnane W, Kerins D, Boylan GB. Heart rate variability during sleep in healthy term newborns in the early postnatal period. Physiol Meas 30: 847-860, 2009. doi:10.1088/0967-3334/30/8/009.

12. Long X, Fonseca P, Aarts RM, Haakma R, Foussier J. Modeling cardiorespiratory interaction during human sleep with complex networks. Appl Phys Lett 105: 203701, 2014. doi:10.1063/1.4902026.

13. Reulecke S, Schulz S, Voss A. Autonomic regulation during quiet and active sleep states in very preterm neonates. Front Physio/ 3: 61, 2012. doi:10.3389/fphys.2012.00061.

14. Sahni R, Schulze KF, Kashyap S, Ohira-Kist K, Myers MM, Fifer WP. Body position, sleep states, and cardiorespiratory activity in developing low birth weight infants. Early Hum Dev 54: 197-206, 1999. doi:10.1016/s0378-3782(98)00104-2.

15. Dick TE, Hsieh YH, Dhingra RR, Baekey DM, Galán RF, Wehrwein E, Morris KF. Cardiorespiratory coupling: common rhythms in cardiac, sympathetic, and respiratory activities. Prog Brain Res 209: 191-205, 2014. doi:10.1016/B978-0-444-63274-6.00010-2.

16. Joshi R, Kommers D, Long X, Feijs L, Van Huffel S, van Pul C, Andriessen P. Cardiorespiratory coupling in preterm infants. J Appl Physiol (1985) 126: 202-213, 2019. doi:10.1152/japplphysiol.00722.2018.

17. Schäfer G, Rosenblum MG, Kurths J, Abel HH. Heartbeat synchronized with ventilation. Nature 392: 239-240, 1998. doi:10.1038/ 32567.

18. Yasuma F, Hayano Jl. Respiratory sinus arrhythmia: why does the heartbeat synchronize with respiratory rhythm? Chest 125: 683690, 2004. doi:10.1378/chest.125.2.683.

19. Coleman WM. On the correlation of the rate of heart beat, breathing, bodily movement and sensory stimuli. J Physio/ 54: 213-217, 1920. doi:10.1113/jphysiol.1920.sp001920.

20. Bartsch RP, Schumann AY, Kantelhardt JW, Penzel T, Ch P. Phase transitions in physiologic coupling. Proc Natl Acad Sci USA 109: 10181-10186, 2012. doi:10.1073/pnas.1204568109.

21. Clark MT, Rusin CG, Hudson JL, Lee H, Delos JB, Guin LE, Vergales BD, Paget-Brown A, Kattwinkel J, Lake DE, Moorman JR. Breath-by-breath analysis of cardiorespiratory interaction for quantifying developmental maturity in premature infants. J Appl Physiol 112: 859-867, 2012. doi:10.1152/japplphysiol.01152.2011.
22. Angelone A, Norman A, Coulter J. Respiratory sinus arrhythmia: a frequency dependent phenomenon. J Appl Physiol 19: 479-482, 1964. doi:10.1152/JAPPL.1964.19.3.479

23. Cysarz D, Bettermann H, Lange S, Geue D, van Leeuwen P. A quantitative comparison of different methods to detect cardiorespiratory coordination during night-time sleep. Biomed Eng Online 3: 44, 2004. doi:10.1186/1475-925X-3-44

24. Hathorn MK. Respiratory sinus arrhythmia in new-born infants. $J$ Physio/ 385: 1-12, 1987. doi:10.1113/jphysiol.1987.sp016480.

25. Thomas RJ, Mietus JE, Peng C-K, Goldberger AL. An electrocardiogram-based technique to assess cardiopulmonary coupling during sleep [Online]. Sleep 28: 1151-1161, 2005. doi:10.1093/sleep/28.9.1151.

26. Patzak A, Lipke K, Orlow W, Mrowka R, Stauss H, Windt E, Persson PB, Schubert E. Development of heart rate power spectra reveals neonatal peculiarities of cardiorespiratory control. Am J Physiol 271: R1025-R1032, 1996. doi:10.1152/ajpregu.1996.271.4.R1025.

27. Rassi D, Mishin A, Zhuravlev YE, Matthes J. Time domain correlation analysis of heart rate variability in preterm neonates. Early Hum Dev 81: 341-350, 2005. doi:10.1016/j.earlhumdev.2004.09.002.

28. Giddens DP, Kitney RI. Neonatal heart rate variability and its relation to respiration [Online]. J Theor Bio/ 113: 759-780, 1985. doi:10.1016/ S0022-5193(85)80192-2.

29. Longin E, Gerstner T, Schaible T, Lenz T, König S. Maturation of the autonomic nervous system: differences in heart rate variability in premature vs. term infants. J Perinat Med 34: 303-308, 2006.

30. Mrowka R, Patzak A, Rosenblum M. Quantitative analysis of cardiorespiratory synchronization in infants. Int J Bifurcation Chaos 10: 2479-2488, 2000. doi:10.1142/S0218127400001754.

31. Lacasa L, Luque B, Ballesteros F, Luque J, Nuño JC. From time series to complex networks: the visibility graph. Proc Natl Acad Sci USA 105: 4972-4975, 2008. doi:10.1073/pnas.0709247105.

32. Mohammadpoory Z, Nasrolahzadeh M, Haddadnia J. Epileptic seizure detection in EEGs signals based on the weighted visibility graph entropy. Seizure 50: 202-208, 2017. doi:10.1016/j.seizure.2017. 07.001 .

33. Wang L, Long $\mathbf{X}$, Arends JBAM, Aarts RM. EEG analysis of seizure patterns using visibility graphs for detection of generalized seizures. $J$ Neurosci Methods 290: 85-94, 2017. doi:10.1016/j.jneumeth.2017. 07.013 .

34. Tang X, Xia L, Liao Y, Liu W, Peng Y, Gao T, Zeng Y. New approach to epileptic diagnosis using visibility graph of high-frequency signal. Clin EEG Neurosci 44: 150-156, 2013. doi:10.1177/1550059412464449.

35. Chen S, Gallagher MJ, Hogg F, Papadopoulos MC, Saadoun S. Visibility graph analysis of intraspinal pressure signal predicts functional outcome in spinal cord injured patients. J Neurotrauma 35: 2947-2956, 2018. doi:10.1089/neu.2018.5775.

36. Prechtl HFR. The behavioural states of the newborn infant (a review). Brain Res 76: 185-212, 1974. doi:10.1016/0006-8993(74) 90454-5.

37. Wijshoff R, Mischi M, Aarts RM. Reduction of periodic motion artifacts in photoplethysmography. IEEE Trans Biomed Eng 64: 196207, 2017. doi:10.1109/TBME.2016.2553060.

38. Albert R, Barabási AL. Statistical mechanics of complex networks. Rev Mod Phys 74: 47-97, 2002. doi:10.1103/RevModPhys.74.47.

39. Watts DJ, Strogatz SH. Collective dynamics of 'small-world' networks. Nature 393: 440-442, 1998. doi:10.1038/30918.

40. Fagiolo G. Clustering in complex directed networks. Phys Rev E Stat Nonlin Soft Matter Phys 76: 026107, 2007.

41. Batalle D, Hughes EJ, Zhang H, Tournier J-D, Tusor N, Aljabar P, Wali L, Alexander DC, Hajnal JV, Nosarti C, Edwards AD, Counsell SJ. Early development of structural networks and the impact of prematurity on brain connectivity. Neuroimage 149: 379-392, 2017. doi:10.1016/j.neuroimage.2017.01.065.

42. Newman MEJ. Assortative mixing in networks. Phys Rev Lett 89 208701, 2002.

43. Newman MEJ, Park J. Why social networks are different from other types of networks. Phys Rev E Stat Nonlin Soft Matter Phys 68 036122, 2003

44. Richman JS, Moorman JR. Physiological time-series analysis using approximate entropy and sample entropy maturity in premature infants physiological time-series analysis using approximate entropy and sample entropy. Am J Physiol Heart Circ Physiol 278: H2O39H2049, 2000. doi:10.1152/ajpheart.2000.278.6.H2039. 
45. Yentes JM, Hunt N, Schmid KK, Kaipust JP, McGrath D, Stergiou $\mathbf{N}$. The appropriate use of approximate entropy and sample entropy with short data sets. Ann Biomed Eng 41: 349-365, 2013. doi:10.1007/s10439-012-0668-3.

46. Lucchini M, Fifer WP, Sahni R, Signorini MG. Novel heart rate parameters for the assessment of autonomic nervous system function in premature infants. Physiol Meas 37: 1436-1446, 2016. doi:10.1088/ 0967-3334/37/9/1436.

47. Werth J, Serteyn A, Andriessen P, Aarts RM, Long X. Automated preterm infant sleep staging using capacitive electrocardiography. Physiol Meas 40: 055003, 2019. doi:10.1088/1361-6579/ab1224.

48. Song C, Havlin S, Makse HA. Origins of fractality in the growth of complex networks. Nature Phys 2: 275-281, 2006. doi:10.1038/ nphys266.

49. Peng CK, Mietus JE, Liu Y, Lee C, Hausdorff JM, Stanley HE, Goldberger AL, Lipsitz LA. Quantifying fractal dynamics of human respiration: age and gender effects. Ann Biomed Eng 30: 683-692, 2002. doi:10.1114/1.1481053.

50. Perkiömäki JS, Mäkikallio TH, Huikuri HV. Fractal and complexity measures of heart rate variability. Clin Exp Hypertens 27: 149-158, 2005.

51. Indic P, Salisbury E, Paydarfar D, Brown E, Barbieri R. Interaction between heart rate variability and respiration in preterm infants. Comput Cardio/ 35: 57-60, 2008. doi:10.1109/CIC.2008.4748976.

52. Ravasz E, Barabási AL. Hierarchical organization in complex networks. Phys Rev E Stat Nonlin Soft Matter Phys 67: 026112, 2003.

53. Lucchini M, Pini N, Fifer W, Burtchen N, Signorini M. Entropy information of cardiorespiratory dynamics in neonates during sleep. Entropy 19: 225, 2017. doi:10.3390/e19050225.

54. Javorka M, Zila I, Balhárek T, Javorka K. Heart rate recovery after exercise: relations to heart rate variability and complexity. Brazilian $J$ Med Biol Res 35: 991-1000, 2002.

55. Porta A, Gnecchi-Ruscone T, Tobaldini E, Guzzetti S, Furlan R, Montano N. Progressive decrease of heart period variability entropy-based complexity during graded head-up tilt. J Appl Physiol (1985) 103: 1143-1149, 2007. doi:10.1152/japplphysiol.00293.2007.

56. Lucchini M, Pini N, Fifer WP, Burtchen N, Signorini MG. Characterization of cardiorespiratory phase synchronization and directionality in late premature and full term infants. Physiol Meas 39: 064001, 2018. doi:10.1088/1361-6579/aac553.

57. Scher MS, Steppe DA, Dokianakis SG, Sun M, Guthrie RD Sclabassi RJ. Cardiorespiratory behavior during sleep in full-term and preterm neonates at comparable postconceptional term ages. Pediatr Res 36: 738-744, 1994. doi:10.1203/00006450-19941200000010.

58. Ravasz E, Somera AL, Mongru DA, Oltvai ZN, Barabási AL. Hierarchical organization of modularity in metabolic networks. Science 297: 1551-1556, 2002. doi:10.1126/science.1073374.

59. Vandersickel N, Van Nieuwenhuyse E, Van Cleemput $\mathbf{N}$ Goedgebeur J, El Haddad M, De NJ, Demolder A, Strisciuglio T, Duytschaever M, Panfilov AV. Directed networks as a novel way to describe and analyze cardiac excitation: directed graph mapping. Front Physiol 10: 1-14, 2019. doi:10.3389/fphys.2019.01138.

60. Werth J, Long X, Zwartkruis-Pelgrim E, Niemarkt H, Chen W, Aarts RM, Andriessen P. Unobtrusive assessment of neonatal sleep state based on heart rate variability retrieved from electrocardiography used for regular patient monitoring. Early Hum Dev 113: 104-113, 2017. doi:10.1016/j.earlhumdev.2017.07.004.

61. Aarts LAM, Jeanne V, Cleary JP, Lieber C, Nelson JS, Bambang Oetomo S, Verkruysse W. Non-contact heart rate monitoring utilizing camera photoplethysmography in the neonatal intensive care unit-a pilot study. Early Hum Dev 89: 943-948, 2013. doi:10.1016/j. earlhumdev.2013.09.016.

62. Gu C, Li C, Lin J, Long J, Huangfu J, Ran L. Instrument-based noncontact doppler radar vital sign detection system using heterodyne digital quadrature demodulation architecture. IEEE Trans Instrum Meas 59: 1580-1588, 2010. doi:10.1109/TIM.2009.2028208.

63. Sekine M, Maeno K. Non-contact heart rate detection using periodic variation in Doppler frequency. SAS 2011-IEEE Sensors Application Symposium Proceedings. San Antonio, TX, USA, 2011. doi:10.1109/ SAS.2011.5739803.

64. Atallah L, Serteyn A, Meftah M, Schellekens M, Vullings R, Bergmans JWM, Osagiator A, Oetomo SB. Unobtrusive ECG monitoring in the NICU using a capacitive sensing array. Physiol Meas 35: 895-913, 2014. doi:10.1088/0967-3334/35/5/895. 